\title{
Randomized controlled trial of lobaplatin plus etoposide vs. cisplatin plus etoposide as first-line therapy in patients with extensive-stage small cell lung cancer
}

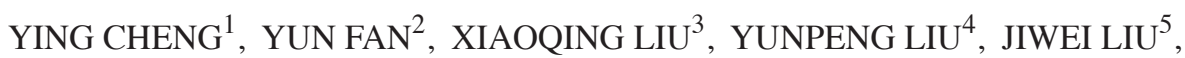 \\ DONG WANG ${ }^{6}$, YAN YU ${ }^{7}$, SHUKUI QIN $^{8}$, WEI LIU ${ }^{9}$, CHENG HUANG $^{10}$, HELONG ZHANG $^{11}$, \\ JUN LIANG $^{12}$, JIANHUA SHI ${ }^{13}$, LIJUN SHENG ${ }^{14}$ and HAO YU ${ }^{15}$
}

${ }^{1}$ Department of Oncology, Jilin Cancer Hospital, Changchun, Jilin 130021; ${ }^{2}$ Department of Oncology, Zhejiang Cancer Hospital, Hangzhou, Zhejiang 310022; ${ }^{3}$ Department of Chest Tumor, 307th Hospital of The Academy of Military Medical Sciences, Beijing 100071; ${ }^{4}$ Department of Oncology, The First Hospital of China Medical University, Shenyang, Liaoning 110001; ${ }^{5}$ Department of Oncology, The First Affiliated Hospital of Dalian Medical University,

Dalian, Liaoning 116011; ${ }^{6}$ Department of Oncology, The Daping Hospital of Third Military Medical University,

Chongqing 400042; ${ }^{7}$ Department of Oncology, The Cancer Hospital of Harbin Medical University, Harbin,

Heilongjiang 150081; ${ }^{8}$ Department of Oncology, People's Liberation Army Cancer Center of Nanjing 81 Hospital,

Nanjing, Jiangsu 210002; ${ }^{9}$ Department of Oncology, Hebei Cancer Hospital, Shijiazhuang, Hebei 050011;

${ }^{10}$ Department of Oncology, Fujian Province Cancer Hospital, Fuzhou, Fujian 350014; ${ }^{11}$ Department of Oncology,

The Tangdu Hospital of Fourth Military Medical University, Xi'an, Shanxi 710038; ${ }^{12}$ Department of Oncology,

Peking University International Hospital, Peking University, Beijing 102206; ${ }^{13}$ Department of Oncology, Linyi Cancer Hospital, Linyi, Shandong 251500; ${ }^{14}$ Department of Oncology, Affiliated Hospital of Shandong Academy of Medical Sciences, Jinan, Shandong 250031; ${ }^{15}$ Department of Biostatistics, Nanjing Medical University, Nanjing, Jiangsu 211166, P.R. China

Received May 18, 2018; Accepted February 4, 2019

DOI: $10.3892 /$ ol.2019.10125

\begin{abstract}
The majority of previous studies of lobaplatin in small cell lung cancer (SCLC) are small phase I-II studies. The present study aimed to verify the non-inferiority (in terms of efficacy) of lobaplatin plus etoposide (EL) vs. cisplatin plus etoposide (EP) in patients with previously untreated extensive-stage SCLC (ES-SCLC). This phase III non-inferiority randomized clinical trial enrolled patients at 17 sites between September 2010 and May 2013. Patients were randomized to EL $\left(30 \mathrm{mg} / \mathrm{m}^{2}\right.$ lobaplatin on day 1 and $100 \mathrm{mg} / \mathrm{m}^{2}$ etoposide on days $1-3$, for 21 -day cycles) or EP $\left(80 \mathrm{mg} / \mathrm{m}^{2}\right.$ cisplatin on day 1 and $100 \mathrm{mg} / \mathrm{m}^{2}$ etoposide on days 1-3, for 21-day cycles). The primary endpoint was progression-free survival (PFS). Secondary endpoints included overall survival (OS), objective response rate, disease control rate (DCR), toxicity and quality of life
\end{abstract}

Correspondence to: Dr Ying Cheng, Department of Oncology, Jilin Cancer Hospital, 1018 Huguang Road, Changchun, Jilin 130021, P.R. China

E-mail: jl.cheng@163.com

Key words: small cell lung cancer, extensive stage, lobaplatin, cisplatin, etoposide, chemotherapy
(QoL). A total of 234 patients were randomized to the EL $(n=122)$ and EP $(n=112)$ treatment groups. The median PFS, median OS and DCR were 5.1 vs. 5.3 months $(\mathrm{P}=0.786)$, 10.6 vs. 9.7 months $(\mathrm{P}=0.701)$ and 85.5 vs. $86.7 \%(\mathrm{P}=0.848)$ in the EL vs. EP groups, respectively. Patients in the EL group had significantly lower frequencies of nephrotoxicity (2.5 vs. $11.7 \% ; \mathrm{P}=0.008)$, nausea (22.3 vs. $40.5 \% ; \mathrm{P}=0.003$ ) and vomiting (14.1 vs. $35.1 \%$; $\mathrm{P}<0.001)$ than those in the EP group. Overall, EL was not inferior to EP in terms of PFS and OS. The tolerance and QoL of the EL regimen were better than those of the EP regimen. EL is thus an alternative choice for the first-line treatment of ES-SCLC.

\section{Introduction}

Lung cancer is the leading cause of tumor-related mortality worldwide (1). Small cell lung cancer (SCLC) accounted for $14 \%$ of lung cancer cases in 2014 (2) and progresses quickly with distant metastasis. Overall, $60-70 \%$ of the patients have extensive-stage SCLC (ES-SCLC) when a definite diagnosis is established (2). Chemotherapy is the primary treatment strategy for ES-SCLC, but the standard first-line therapeutic regimens for SCLC, typically platinum (cisplatin/carboplatin) combined with topoisomerase inhibitors, achieve a median survival time of 8-13 months only (2), with high toxicity including myelosuppression, gastrointestinal toxicity, cardiac toxicity and nephrotoxicity $(3,4)$. 
Lobaplatin is a third-generation platinum drug, discovered during studies of platinum compounds for cisplatin-resistant tumors. Pre-clinical studies showed that compared with cisplatin, lobaplatin has equivalent activity against tumors (5-7), but with improved tolerability and stability, and lower toxicity $(8,9)$. A phase II trial further suggested that lobaplatin had strong antitumor activity in patients with SCLC who were naïve or previously treated with one line of therapy (10). The majority of previous studies were limited in sample size and restricted to phase II trials, limiting the reliable assessment of the regimen $(11,12)$.

Therefore, the aim of the present study was to verify the non-inferiority (in terms of efficacy) of lobaplatin plus etoposide (EL) vs. cisplatin plus etoposide (EP) in patients with previously untreated ES-SCLC.

\section{Materials and methods}

Study design. The present study was a non-inferiority, open label, randomized clinical trial (Chinese Clinical Trial Registry ChiCTR-TRC-10001047) that was performed at 17 sites in China between September 2010 and May 2013. The study was approved by the ethical committees of all participating hospitals, and followed the Good Clinical Practice (GCP) principle and Declaration of Helsinki. No substantial protocol revision was made during study implementation. All patients provided written informed consent prior to enrollment.

Patients. The inclusion criteria were as follows: i) Definitive histological diagnosis of ES-SCLC (defined as a tumor that spreads beyond the hemithorax, hilar, mediastinal or supraclavicular nodes); ii) an age of 18-70 years; iii) no chemotherapy history; iv) palliative radiotherapy or surgery received for metastatic lesions, completed for $>14$ days; v) measurable lesions (non-irradiated sites); according to the Response Evaluation Criteria in Solid Tumors (RECIST, version 1.1) (13), patients with lesions with a diameter $\geq 20 \mathrm{~mm}$ by plain computed tomography (CT) or $\geq 10 \mathrm{~mm}$ by helical $\mathrm{CT}$, and the maximum length of $>2$ times the section thickness; vi) an Eastern Cooperative Oncology Group performance status (ECOG-PS) (14) of 0-1; and vii) an estimated survival time of $>3$ months.

The exclusion criteria were as follows: i) A previous history of allergy to platinum compounds; ii) an active ulcer; iii) surgery or radiotherapy for the primary lesion; iv) interstitial pneumonia or pulmonary fibrosis; v) active brain metastasis and a stable status for $<4$ weeks and/or with symptoms, and/or requiring anticonvulsant drugs or steroids and/or treatment for leptomeningeal disease; vi) severe main bronchial or lobe bronchial stenosis and obstruction caused by tumor invasion or oppression, or presenting with superior vena cava syndrome (SVCS), or with uncontrolled malignant hydrothorax, ascites or pericardial effusion at an above medium degree; vii) severe infection, severe anti-diuretic hormone abnormal secretion syndrome, poorly controlled diabetes mellitus (requirement of continuous daily insulin of $>40$ units, or fasting plasma glucose remaining $>7.8 \mathrm{mmol} / 1$ and hemoglobin A1c $>9.0 \%$ with or without continuous daily insulin of $<40$ units), or with severe complications of treatment-required SVCS; viii) severe cardiovascular diseases, including high blood pressure uncontrollable by medications, unstable angina, myocardial infarction attack in the past 6 months, congestive heart failure of New York Heart Association (15) grade $>3$, or severe arrhythmias; ix) requirement for long-term anticoagulants or vitamin $\mathrm{K}$ antagonists, including warfarin, heparin or its analogues, except for prophylactic low-dose warfarin ( $\leq 1 \mathrm{mg} /$ day) or aspirin (100 mg/day); $\mathrm{x})$ participated in another clinical trial within 4 weeks of allocation, or had quit this study following allocation; xi) active cancer relapse, except for intraepithelial carcinoma, or no recurrence of relapsed cancer for >5 years; or xii) pregnancy, possible pregnancy or an intention for pregnancy, a lack of effective contraceptive measures or currently lactating.

Randomization. All enrolled patients were assigned at a 1:1 ratio to receive EL or EP. Randomization was implemented using a central computerized randomization system managed by an independent statistician. Randomization was stratified according to ECOG PS, sex, age, and presence or absence of liver or brain metastasis.

Treatment protocol. According to phase I studies, the recommended dose of lobaplatin is $50 \mathrm{mg} / \mathrm{m}^{2}$ as single-agent chemotherapy $(16,17)$. In a phase I study (Clinical Trial Approval Number: 2008L09400), the maximum tolerated dose for the lobaplatin combination regimen as first-line therapy in patients with non-small cell lung cancer (NSCLC) was $30 \mathrm{mg} / \mathrm{m}^{2}$ lobaplatin on day 2 and $175 \mathrm{mg} / \mathrm{m}^{2}$ paclitaxel on day 1 of a 21-day treatment cycle. Therefore, $30 \mathrm{mg} / \mathrm{m}^{2}$ lobaplatin was selected in the present study.

The EL regimen was composed of six cycles of $30 \mathrm{mg} / \mathrm{m}^{2}$ lobaplatin (10 mg/vial; National Medical Authorization No. H20080359; Hainan Changan International Pharmaceutical Co., Ltd, Haikou, Hainan, China) on day 1 and $100 \mathrm{mg} / \mathrm{m}^{2}$ etoposide (100 mg/vial; National Medical Authorization No. H37023183; Qilu Pharmaceutical Co., Ltd, Jinan, Shandong, China) on days 1-3. The EP regimen consisted of six cycles of $80 \mathrm{mg} / \mathrm{m}^{2}$ cisplatin (10 mg/vial; National Medical Authorization No. H37021358; Qilu Pharmaceutical Co., Ltd, Jinan, Shandong, China) on day 1 and $100 \mathrm{mg} / \mathrm{m}^{2}$ etoposide on days 1-3. The cycle length was 21 days for each arm.

If a patient presented with hematological toxicities [platelets $<25 \times 10^{9} / 1$ or absolute neutrophil count (ANC) $<0.5 \times 10^{9} / 1$ for 4 consecutive days, or ANC $<1.0 \times 10^{9} / 1$ combined with a fever of $>38.5^{\circ} \mathrm{C}$ during the prior course], the doses of lobaplatin and etoposide were reduced by 10 and $20 \mathrm{mg} / \mathrm{m}^{2}$, respectively, in the subsequent cycles. A $25 \%$ dose reduction of cisplatin was required when grade $>2$ neurotoxicity or nephrotoxicity occurred. Once the dose was reduced, the new dose was kept for the subsequent cycles and there was no return to the original dose. The dose of etoposide could be reduced a maximum of two times (i.e. reduced to $60 \mathrm{mg} / \mathrm{m}^{2}$ ). If grade $3 / 4$ toxicity still occurred following two dose adjustments, the treatment was terminated and the patient was withdrawn from the study.

Any radiotherapy or other antitumor therapy was forbidden until disease progression. Chinese medicine and immunomodulatory agents with explicit lung cancer treatment indications that were approved by the State Food and Drug Administration were not permitted for concomitant administration. Patients were administered the antiemetic prophylaxis and treatments 
according to the routine medical practice at each participating center. The recommended prophylactic antiemetic regimen was a 5-hydroxytryptamine receptor 3 antagonist in combination with glucocorticoids and, if necessary, in combination with other antiemetics or sedatives. When patients presented with grade 3/4 thrombocytopenia, thrombopoietin (TPO) or interleukin (IL)-11 could be used. If the patients developed bone metastases, bisphosphonates could be used. Hospitals were allowed to provide any relevant supportive and symptomatic measures according to their routine medical care practice.

Treatment assessments. During treatment, physical examination, electrocardiogram, blood coagulation tests, tumor marker tests and urinalysis were performed within 1 week of each cycle. Blood count and blood biochemistry analyses were performed at least once a week. Spiral CT scan or magnetic resonance imaging was used for tumor assessment according to RECIST (version 1.1) at baseline (within 2 weeks of treatment) and once every two cycles following treatment (the 21st day of the second cycle, the 21st day of the 4th cycle and the 21 st day of the 6 th cycle, \pm 3 day window), until progressive disease (PD) was diagnosed or the patient withdrew from the study. Toxicity was evaluated according to the Common Terminology Criteria for Adverse Events (18) (CTC AE, version 3.0). Quality of life (QoL) was assessed using EuroQol (EQ)-5D, EQ-visual analogue scales (VAS), QoL questionnaire (QLQ)-C30 (version 3.0) (19) and QLQ-lung cancer 13 (QLQ-LC13) (20), all of which were collected on day 1 of each cycle. Questionnaires were filled in by the patients under the instruction of nurses from each study center who received specific and GCP training.

Endpoints. Progression-free survival (PFS) referred to the time interval from randomization to disease progression or mortality from any cause. In cases where the information on mortality or disease progression was not available, PFS referred to the time interval between randomization and the last follow-up.

Overall survival (OS) referred to the time interval from randomization to mortality from any cause. In cases where the information on mortality was not available, OS referred to the time interval between randomization and the last follow-up.

The overall response rate (ORR) referred to the ratio of cases with optimal efficacy [complete remission $(\mathrm{CR})+$ partial remission (PR)] vs. the total cases. The disease control rate (DCR) referred to the ratio of cases with $\mathrm{CR}, \mathrm{PR}$ and stable disease (SD) [namely $\mathrm{CR}+\mathrm{PR}+\mathrm{SD}$ ( $\geq 8$ weeks)], vs. the total cases.

The QoL variables originated from the QLQ-C30, QLQ-LC13, EQ-5D and EQ-VAS. QLQ-C30 comprises 30 items organized into five functional scales, three symptom scales, one overall health/quality-of-life scale, and six single items. QLQ-LC13 is a supplementary questionnaire for patients with lung cancer and includes questions assessing cough, hemoptysis, dyspnea, site-specific pain, treatment-associated side effects and the efficacy of pain medications. The EQ-5D records the level of self-reported problems according to five dimensions. EQ-VAS is a VAS used by respondents to describe their own health from 0 (the worst imaginable health) to 100 (the best imaginable health). The raw score (RS) for one area was calculated by the sum of scores from every item under the corresponding area divided by the number of items. The standard score (SS) was calculated using the following formula: $\mathrm{SS}=[1-(\mathrm{RS}-1) / \mathrm{R}] \mathrm{x} 100$.

The primary endpoint was PFS. The secondary endpoints included OS, ORR, DCR, toxicity and QoL. Adverse events (AEs) taking place during the trial were documented and reviewed for all patients.

Statistical analysis. The primary analysis of this study aimed to demonstrate the non-inferiority of EL to EP with regard to PFS. The clinical non-inferiority margin for the hazard ratio (HR) was set at 1.4 based on the primary objective of this study, current treatment, prognosis of the disease and historical data. According to the literature $(21,22)$ and the experience of the investigators, the median PFS (mPFS) time in the EL group and the EP group was considered to be 5.0 months. Taking two-sided $\alpha=0.05$ and $1-\beta=80 \%$, and considering a drop-out rate of $10 \%$ in each group (patient enrollment was planned as 24 months, followed by 18 months of follow-up), this clinical trial required a total of 234 patients $(23,24)$.

Interim analysis was not preset, and the termination was $80 \%$ PD events. Normally distributed continuous variables are presented as the mean \pm standard deviation. Non-normally distributed continuous variables are presented as the median (range). Categorical variables are presented as frequencies and percentages. Comparisons of baseline demographics and disease characteristics between groups were made using Student's t-test for continuous variables and the $\chi^{2}$ test for categorical variables. The survival curve of PFS was analyzed by log-rank test, and the HR and confidence interval (CI) of PFS were calculated by Cox proportional hazards regression model. The Cox proportional hazards regression model incorporating covariates, including sex (males vs. females), age ( $\geq 65$ vs. $<65$ years), stage (stage IV vs. IIIB) and ECOG PS ( 1 vs. 0), was used to estimate HR and its $95 \%$ CI. The Kaplan-Meier method was used to estimate the mPFS and mOS times, and survival curves were plotted. Fisher's exact test was used for the comparison of the AEs. The Wilcoxon rank sum test was used for comparison of changes in the QoL scores prior to and after treatment between the two groups. Statistical analyses were conducted using SAS 9.2 (SAS Institute, Cary, NY, USA). Two-sided P-values of $<0.05$ were considered to indicate a statistically significant difference.

\section{Results}

Enrollment. A total of 234 were enrolled: 122 in the EL group and 112 in the EP group. In the EL group, 1 patient was finally pathologically diagnosed with NSCLC, and 1 patient in the EP group withdrew consent prior to treatment, but following randomization. Overall, 232 patients were included in the intention-to-treat analysis. In the EL group, 3 patients violated the protocol and 1 withdrew consent during treatment. In the EP group, 1 patient violated the protocol, 2 withdrew consent and 3 were lost to follow-up (Fig. 1). Finally, 222 patients strictly adhered to the protocol (117 in the EL group and 105 in the EP group) and were included in the per-protocol analysis. Baseline characteristics were similar between the two groups (Table I). 
Table I. Baseline characteristics of the patients.

\begin{tabular}{|c|c|c|c|}
\hline Variable & $\mathrm{EL}(\mathrm{n}=121)$ & $\mathrm{EP}(\mathrm{n}=111)$ & P-value \\
\hline \multicolumn{4}{|l|}{ Age } \\
\hline Mean \pm standard deviation, years & $56.3 \pm 8.0$ & $56.4 \pm 8.4$ & 0.952 \\
\hline$\geq 65$ years, $\mathrm{n}(\%)$ & $19(15.7)$ & $18(16.2)$ & 0.481 \\
\hline Sex, n $(\%)$ & & & 0.878 \\
\hline Male & $93(76.9)$ & $84(75.7)$ & \\
\hline Female & $28(23.1)$ & $27(24.3)$ & \\
\hline ECOG, n (\%) & & & 0.251 \\
\hline 0 & $13(10.7)$ & $7(6.3)$ & \\
\hline 1 & $108(89.3)$ & $104(93.7)$ & \\
\hline Affected lung, n (\%) & & & 0.429 \\
\hline Left & $52(43.0)$ & $54(48.6)$ & \\
\hline Right & $68(56.2)$ & $57(51.4)$ & \\
\hline Both & $1(0.8)$ & $0(0.0)$ & \\
\hline History of smoking, n (\%) & & & 0.460 \\
\hline Non-smokers & $35(28.9)$ & $27(24.3)$ & \\
\hline Smokers & $86(71.1)$ & $84(75.7)$ & \\
\hline TNM stage, n (\%) & & & 0.318 \\
\hline IIIB & $3(2.5)$ & $6(5.4)$ & \\
\hline IV & $118(97.5)$ & 105 (94.6) & \\
\hline Liver/brain metastasis, n (\%) & & & 0.791 \\
\hline With & $49(40.5)$ & $47(42.3)$ & \\
\hline Without & $72(59.5)$ & $64(57.7)$ & \\
\hline Bone metastasis, $\mathrm{n}(\%)$ & & & 0.236 \\
\hline With & $27(22.3)$ & $32(28.8)$ & \\
\hline Without & $94(77.7)$ & $79(71.2)$ & \\
\hline History of malignant tumor, n (\%) & & & 0.248 \\
\hline No & $118(97.5)$ & $111(100.0)$ & \\
\hline Yes & $3(2.5)$ & $0(0.0)$ & \\
\hline Other major health problems, $\mathrm{n}(\%)$ & & & 0.674 \\
\hline No & $84(69.4)$ & $74(66.7)$ & \\
\hline Yes & $37(30.6)$ & $37(33.3)$ & \\
\hline
\end{tabular}

ECOG, Eastern Cooperative Oncology Group; EL, lobaplatin plus etoposide; EP, cisplatin plus etoposide; TNM, Tumor-Node-Metastasis (30).

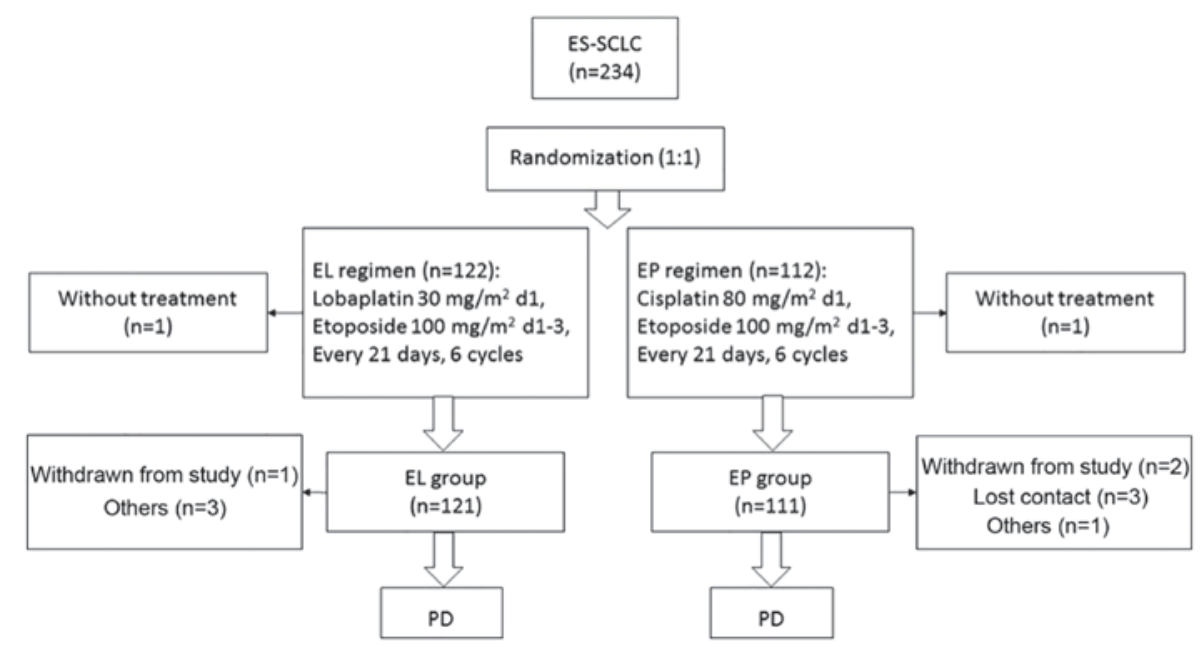

Figure 1. Trial flowchart. ES-SCLC, extensive stage of small cell lung cancer; EL, lobaplatin plus etoposide; EP, cisplatin plus etoposide; PD, progressive disease; d, day. 


\section{A Progression-free survival}

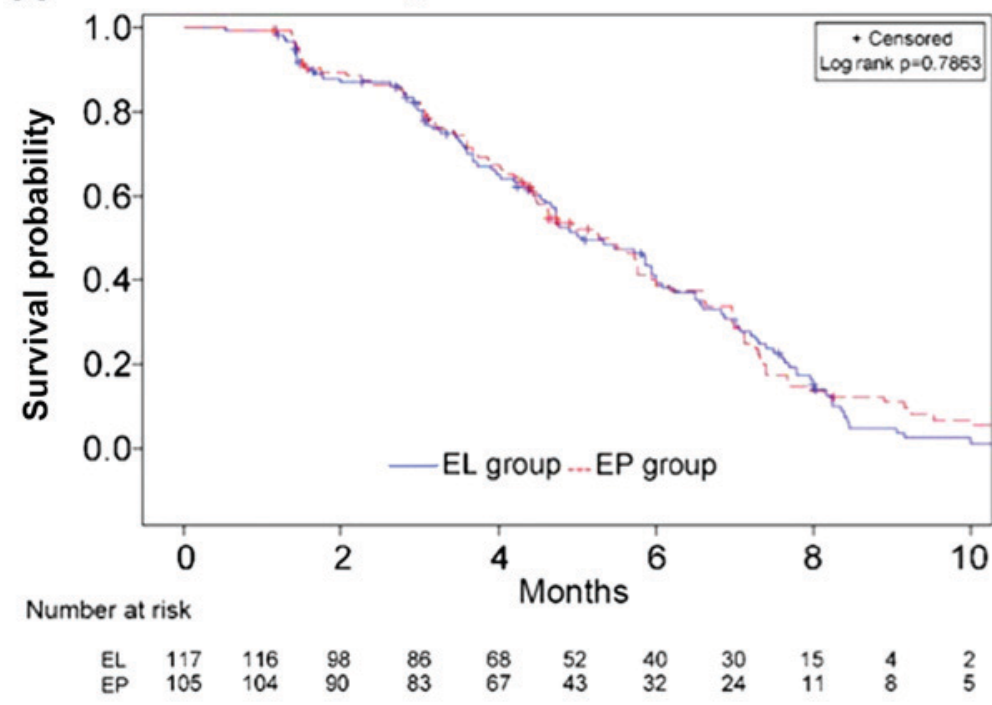

B Overall survival

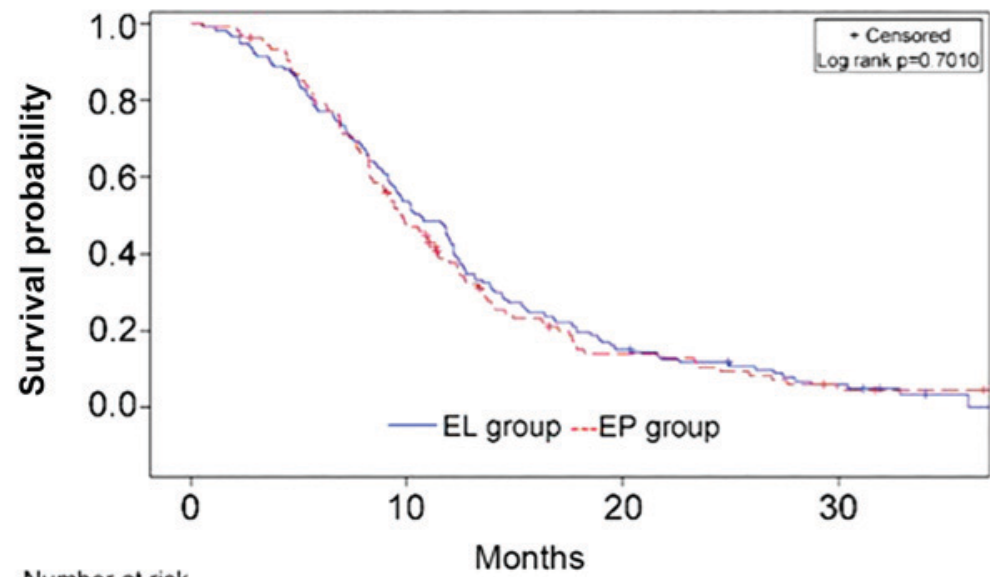

Number at risk

$\begin{array}{llllllllllllllllllll}\text { EL } & 117 & 113 & 104 & 90 & 79 & 63 & 51 & 36 & 29 & 23 & 18 & 14 & 13 & 11 & 8 & 6 & 3 & 2 & 1 \\ \text { EP } & 105 & 104 & 97 & 82 & 69 & 48 & 35 & 24 & 21 & 13 & 12 & 11 & 9 & 7 & 5 & 3 & 2 & 2 & 2\end{array}$

Figure 2. Progression-free survival and overall survival in the EL and EP groups. (A) Progression-free survival in the EL and EP groups. (B) Overall survival in the EL and EP groups. EL, lobaplatin plus etoposide; EP, cisplatin plus etoposide.

Efficacy. The median PFS time in the EL $(\mathrm{n}=117)$ and EP $(\mathrm{n}=105)$ groups was 5.1 months (range, 1.3-16.3 months) and 5.3 months (range, 1.1-13.8 months), respectively (HR, 1.041; 95\%CI, 0.777-1.391; $\mathrm{P}=0.786$ ) (Fig. 2A). The median OS time in the EL and EP groups was 10.6 vs. 9.7 months, respectively (HR, 0.947; 95\%CI, 0.719-1.248; $\mathrm{P}=0.701$ ) (Fig. 2B). There were no differences when stratifying by age, sex, ECOG PS and presence/absence liver/brain metastases (Fig. 3). The two groups did not exhibit any significant difference in ORR (67.6 vs. $53.9 \% ; \mathrm{P}=0.051)$ or $\mathrm{DCR}(86.7$ vs. $85.5 \% ; \mathrm{P}=0.850$ ).

Toxicity. Toxicity analysis was conducted among 232 patients who received treatment and the common treatment-emergent AEs are summarized in Table II. There were 54 (44.6\%) patients in the EL group and 77 (69.4\%) patients in the EP group who experienced AEs $(\mathrm{P}<0.001)$. In terms of Grade $3 / 4$ AEs, the EL group had a significantly lower frequency than the EP group (5.8 vs. $15.3 \% ; \mathrm{P}=0.019)$. Renal toxicity, nausea, vomiting, loss of appetite, hiccup, fatigue and particularly Grade $3 / 4$ (11.7 vs. $0.8 \%$; $\mathrm{P}<0.001$ ) were more common in the EP group.
The two groups did not have significant differences in the frequency and severity of anemia, leucopenia or neutropenia, although the EL group had a significantly higher frequency of Grade 3/4 thrombocytopenia than the EP group (28.9 vs. $10.8 \% ; \mathrm{P}=0.001)$. Due to thrombocytopenia, 5 patients in the EL group and 1 patient in the EP group required a 6- to 10-day delay prior to the following cycle. A total of 18 patients (14.9\%) in the EL group and 8 (7.2\%) in the EP group received IL-11 or a platelet transfusion $(\mathrm{P}=0.090)$. No bleeding was observed in either group. The two groups had a similar frequency of serious AEs (6.6 vs. $6.3 \% ; \mathrm{P}=1.000)$.

QoL. According to the QLQ-C30, the EL group had significantly better outcomes in terms of nausea/vomiting and fatigue at various time points following chemotherapy compared with the EP group $(\mathrm{P}<0.05)$. With regard to the QLQ-LC13, the EL group had significantly better outcomes for alopecia and shortness of breath at various time points following the second treatment cycle compared with the EP group $(\mathrm{P}<0.05)$ (Fig. 4). The two groups did not show significant differences in 

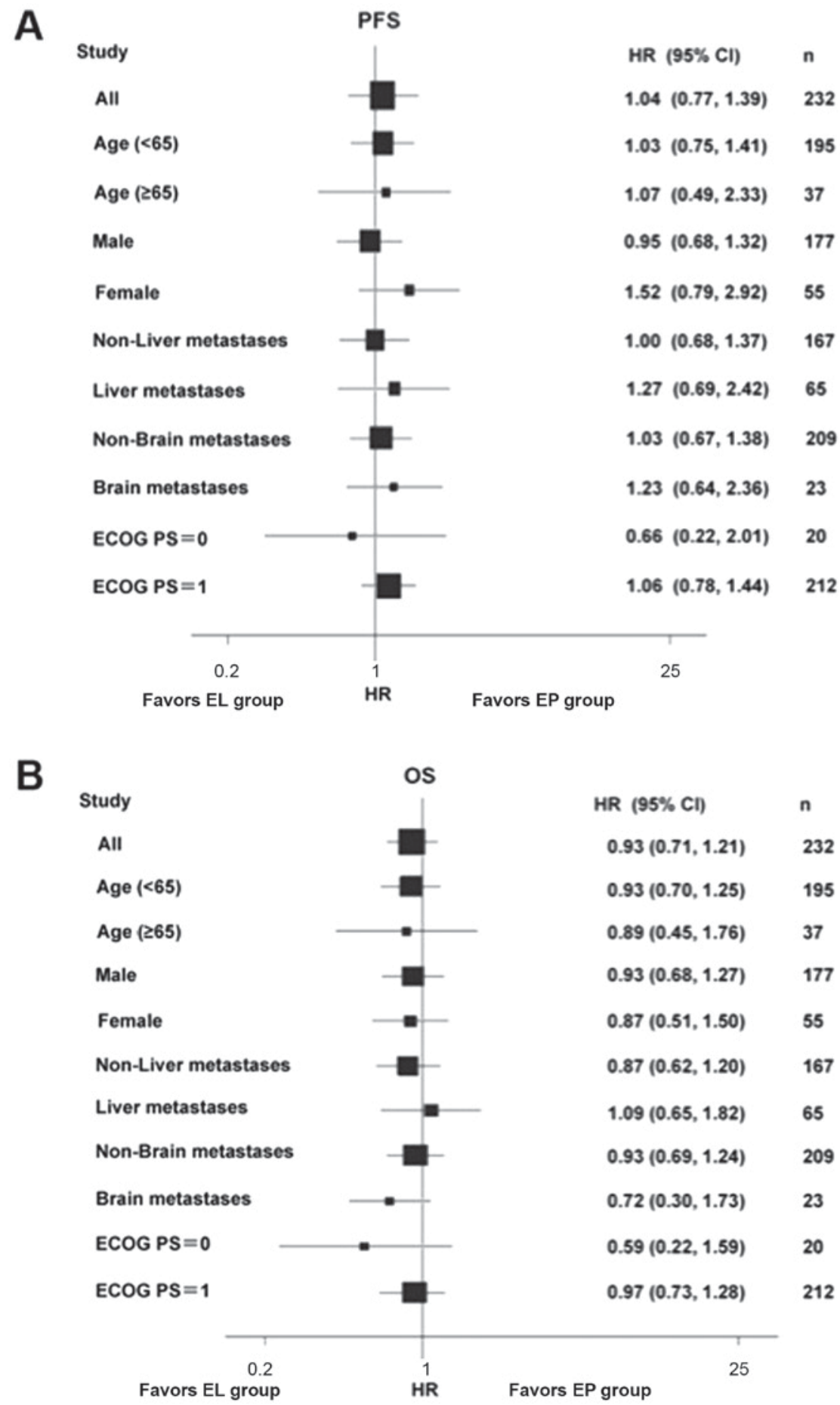

Figure 3. Forest plot of PFS and OS. (A) Forest plot of PFS. (B) Forest plot of OS. PFS, progression-free survival; OS, overall survival; ECOG PS, Eastern Cooperative Oncology Group performance status; EL, lobaplatin plus etoposide; EP, cisplatin plus etoposide; HR, hazard ratio; CI, confidence interval.

patient-reported outcomes and other QoL indicators $(\mathrm{P}>0.05)$. Prior to treatment, patients in the two groups achieved varying degrees of improvement in their QoL scores, particularly with regard tonausea/vomiting $(\mathrm{P}<0.001)$, loss of appetite $(\mathrm{P}<0.001$; data not show) and fatigue $(\mathrm{P}<0.001)$.

Concomitant medications. The proportion of patients in the EL group who received concomitant medications was significant lower than that in the EP group in terms of diuretic agents (36.4 vs. $100 \%$; $\mathrm{P}<0.001$ ), renal protective agents ( 8.3 vs. $24.3 \%$; $\mathrm{P}=0.001)$, gastric mucosa protective agents (57.0 vs. $71.1 \%$;
$\mathrm{P}=0.029)$, electrolytes (28.1 vs. $42.2 \%$; $\mathrm{P}=0.028$ ) and vitamins ( 22.3 vs. $35.1 \% ; \mathrm{P}=0.041)$. There were no significant differences between the two groups in other concomitant medications.

Subsequent antitumor therapy. Once they presented with $\mathrm{PD}, 51(42.1 \%)$ patients in the EL group received second-line chemotherapy, of which 14 (11.6\%) patients received both second-line chemotherapy and local palliative radiotherapy, with $8(6.6 \%)$ patients only receiving local palliative radiotherapy, 8 (6.6\%) patients receiving other therapies, 12 (9.9\%) patients without information on subsequent treatment and 
Table II. Adverse events in the EL and EP groups.

\begin{tabular}{|c|c|c|c|c|c|c|}
\hline \multirow[b]{2}{*}{ Variable, n (\%) } & \multicolumn{2}{|c|}{ EL } & \multicolumn{2}{|c|}{ EP } & \multicolumn{2}{|c|}{ P-value } \\
\hline & Any grade & Grade III/IV & Any grade & Grade III/IV & Any grade & Grade III/IV \\
\hline All events & 54 (44.6) & $7(5.8)$ & 77 (69.4) & $17(15.3)$ & $<0.001$ & 0.019 \\
\hline Renal toxicity & $3(2.5)$ & - & $13(11.7)$ & - & 0.006 & - \\
\hline Elevated bilirubin & $30(24.8)$ & $11(9.1)$ & $41(36.9)$ & 13 (11.7) & 0.048 & 0.527 \\
\hline Nausea & $27(22.3)$ & $1(0.8)$ & $45(40.5)$ & $3(2.7)$ & 0.003 & 0.351 \\
\hline Vomiting & $17(14.0)$ & $1(0.8)$ & $39(35.1)$ & $13(11.7)$ & $<0.001$ & $<0.001$ \\
\hline Loss of appetite & $8(6.6)$ & - & $18(16.2)$ & - & 0.003 & - \\
\hline Hiccup & $0(0.0)$ & - & $7(6.3)$ & - & 0.005 & - \\
\hline Fatigue & $13(10.7)$ & $1(0.8)$ & $25(22.5)$ & $1(0.9)$ & 0.020 & 1.000 \\
\hline Leucopenia & $108(89.3)$ & $41(33.9)$ & $99(89.2)$ & $37(33.3)$ & 1.000 & 1.000 \\
\hline Neutropenia & $95(78.5)$ & $78(64.5)$ & $95(85.6)$ & $82(73.9)$ & 0.176 & 0.155 \\
\hline Anemia & $96(79.3)$ & $28(23.1)$ & $87(78.4)$ & $17(15.3)$ & 0.873 & 0.139 \\
\hline Thrombocytopenia & $63(52.1)$ & $35(28.9)$ & $46(41.4)$ & $12(10.8)$ & 0.115 & 0.001 \\
\hline
\end{tabular}

EL, lobaplatin plus etoposide; EP, cisplatin plus etoposide.

$42(34.7 \%)$ patients without any antitumor therapy. In the EP group, the corresponding figures were 38 (34.2\%), where 7 patients received both second-line chemotherapy and local palliative radiotherapy, and $9(8.1 \%), 2(1.8 \%), 5(4.5 \%)$ and $57(51.4 \%)$ with the respective aforementioned conditions; the differences in numbers were significant $(\mathrm{P}=0.028)$.

\section{Discussion}

This phase III non-inferiority randomized clinical trial aimed to verify the non-inferiority (in terms of efficacy) of EL vs. EP in patients with previously untreated ES-SCLC. The results showed that EL is not inferior to EP in terms of PFS and OS. The tolerance and QoL of the EL regimen were greater than that for the EP regimen. Therefore, EL is an alternative choice for the first-line treatment of ES-SCLC in China.

A significant finding of the present study is that the EL regimen, as first-line therapy for ES-SCLC, reduced the frequency of non-hematological AEs. Renal toxicity of cisplatin, which is dose-limiting, has a frequency of up to 20-30\% (25). Although hydration and application of diuretics aid in reducing the occurrence, the frequency increases with dosage accumulation. In the present study, the frequency of renal toxicity associated with EL was only $2.5 \%$ and mostly grade $1 / 2$, suggesting that EL has less renal toxicity, which was also confirmed by a study using lobaplatin plus paclitaxel to treat esophageal squamous carcinoma (26). Given the median onset age of SCLC, the decreased renal reserve function with age and renal impairment resulting from possible complications, lobaplatin may be a more appropriate treatment option for elderly SCLC patients with renal dysfunction, but this will have to be validated due to the small number of patients $>65$ years old in the present study and as the patients were not analyzed in terms of renal function. Gastrointestinal AEs, including nausea and vomiting, are additional considerations that restrict the clinical use of cisplatin. In the present study, the EL group had a significantly lower frequency of grade 3/4 nausea and vomiting than the EP group. Similar results were also found in other studies of lobaplatin-based chemotherapy regimens (26-28), although the types of tumors were different. Therefore, the EL regimen is possibly an ideal option for SCLC patients who are clinically intolerant to the gastrointestinal AEs of cisplatin.

In the present study, the EL group had a higher frequency of thrombocytopenia than the EP group, but the majority of the patients recovered within 2 weeks. The EL group, in comparison with the EP group, did not show statistical differences in the frequency and severity of leucopenia, neutropenia or anemia. Thrombocytopenia is a dose-limiting toxicity of lobaplatin, and its severity is associated with the dose of lobaplatin and level of creatinine clearance (Ccr) (16). A study showed that the maximum tolerated dose of lobaplatin was $40 \mathrm{mg} / \mathrm{m}^{2}$ in a population with a Ccr of $60-80 \mathrm{ml} / \mathrm{min}, 70 \mathrm{mg} / \mathrm{m}^{2}$ in a population with a Ccr of $81-100 \mathrm{ml} / \mathrm{min}$ and $85 \mathrm{mg} / \mathrm{m}^{2}$ in a population with a $\mathrm{Ccr}>100 \mathrm{ml} / \mathrm{min}$; the median time to occurrence of thrombocytopenia was 10 days (range, 7-14 days), and the median time to recovery was 7 days (range, 2-18 days) (17). Therefore, determining the individual dosage of lobaplatin according to the serum Ccr of the patient is an effective measure to prevent the occurrence of severe thrombocytopenia. Furthermore, coagulation routine examination should be performed prior to receiving the lobaplatin-containing regimen. Caution should be taken when using the EL regimen in patients with SCLC and coagulation disorders or bleeding events within 4 weeks prior to treatment.

Another important finding is that the EL regimen was superior to the EP regimen in terms of improving QoL. As ES-SCLC is an incurable disease, improving QoL is a common goal pursued by the patient and the physician in addition to prolonging survival. In the present study, EQ-5D and EQ-VAS, QLQ-C30 and QLQ-LC13 were used to analyze 

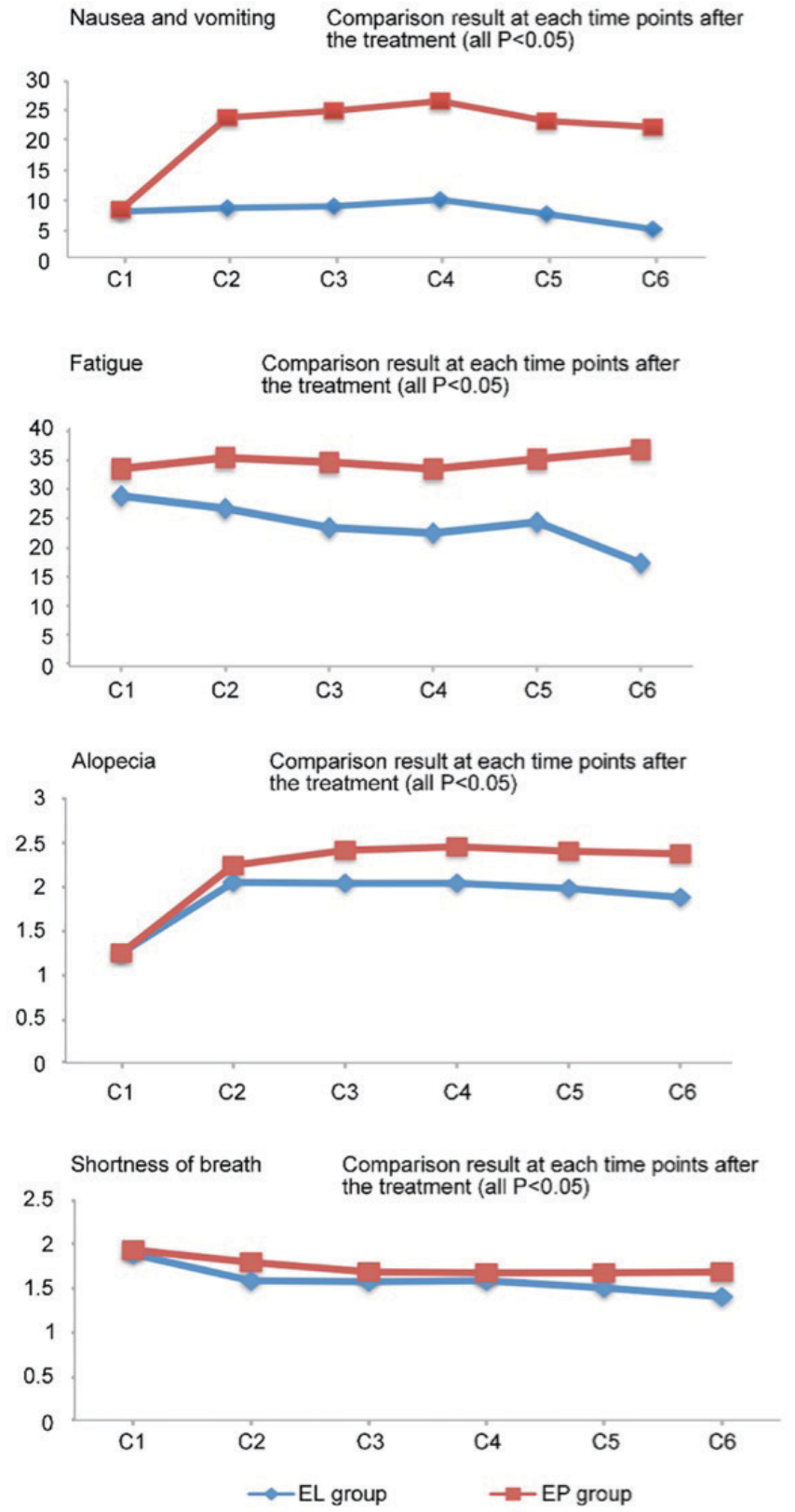

Figure 4. Comparison of quantitative quality of life scores (nausea and vomiting, fatigue, alopecia and shortness of breath) at various time points between the EL and EP groups. EL, lobaplatin and etoposide; EP, cisplatin and etoposide.

and evaluate the patients' QoL. The results showed that the EL group achieved more significant improvements in fatigue, nausea/vomiting and loss of appetite than the EP group. These results are supported by a trial in patients with cervical cancer (29). Along with improvements in survival, the patients in the EL group showed greater compliance with treatment, possibly due a more tolerable AE profile of the EL regimen. In addition, the better tolerability of the EL regimen could possibly make the patients fitter to receive subsequent lines of therapy, making improvements in survival possible. In the present study, more patients received treatments following PD. However, as those subsequent treatments were administered outside the protocol, data were scarce and QoL was not evaluated. This will require assessment to be examined in a future study.
Finally, it is noteworthy that the cost of lobaplatin is 10-12 times higher compared with cisplatin, as it is still in the patent protection period in China. Nevertheless, the lobaplatin-based regimen has more advantages in reducing concomitant medications compared with the cisplatin-based regimen. In addition, the overall cost of lobaplatin-based regimen will be reduced following the patent expiration of lobaplatin.

The present study is not without limitations. Despite the fact that it was a multicenter study, the sample size was relatively small and all patients were Chinese. Differences in cancer genetics among populations may preclude the generalizability of the present trial and confirmation in other populations is required. Nevertheless, the present trial shows promising results for the treatment of SCLC and the applicability of the EL regimen.

In conclusion, for ES-SCLC treatment-naïve patients, the EL regimen was not inferior to the EP regimen in terms of efficacy in the present study. In addition, the EL regimen had a significantly lower overall frequency and severity of AEs compared with the EP regimen. The EL regimen may offer an alternative first-line therapy for patients with ES-SCLC. Meanwhile, further research is necessary to evaluate the efficacy and safety of EL in the treatment of SCLC in larger patient populations, including elderly patients and patients with renal dysfunction.

\section{Acknowledgements}

This abstract was presented at the American Society of Clinical Oncology Annual Meeting, May 30-June 3, 2014 in Chicago, IL, USA and was published as Abstract no. 7595 in Journal of Clinical Oncology 32 (Suppl 15) 2014.

\section{Funding}

This study was funded by the China State Project for Essential Drug Research and Development (grant no. 2013ZX09104001).

\section{Availability of data and materials}

The datasets used and/or analyzed during the current study are available from the corresponding author on reasonable request.

\section{Authors' contributions}

YC, YF, XL, YL, JWL, DW, YY, SQ, WL, CH, HZ, JL, JS and LS performed the studies, participated in collecting data and offering suggestions for revision of the manuscript. HY performed the statistical analysis and participated in its design. All authors read and approved the final manuscript.

\section{Ethics approval and consent to participate}

The study was approved by the ethical committees of following affiliations: Jilin Cancer Hospital, Changchun, Jilin; Zhejiang Cancer Hospital, Hangzhou, Zhejiang; 307th Hospital of the Academy of Military Medical Sciences, Beijing; The First Hospital of China Medical University, Shenyang, Liaoning; The First Affiliated Hospital of Dalian Medical University, 
Dalian, Liaoning; The Daping Hospital of Third Military Medical University, Chongqing; The Cancer Hospital of Harbin Medical University, Harbin, Heilongjiang; People's Liberation Army Cancer Center of Nanjing 81 Hospital, Nanjing, Jiangsu; Hebei Cancer Hospital, Shijiazhuang, Hebei; Fujian Province Cancer Hospital, Fuzhou, Fujian; The Tangdu Hospital of Fourth Military Medical University, Xi'an, Shanxi; Peking University International Hospital, Peking University, Beijing; Linyi Cancer Hospital, Linyi, Shandong; Affiliated Hospital of Shandong Academy of Medical Sciences, Jinan, Shandong and followed the Good Clinical Practice principle and Declaration of Helsinki. No substantial protocol revision was made during study implementation. All patients provided written informed consent prior to enrollment.

\section{Patient consent for publication}

Not applicable.

\section{Competing interests}

The authors declare that they have no competing interests.

\section{References}

1. Howlader N, Noone A and Krapcho M: SEER cancer statistics review, 1975-2013, based on November 2015 SEER data submission, posted to the SEER web site, April 2016. Bethesda: National Cancer Institute, 2016.

2. Pietanza MC, Byers LA, Minna JD and Rudin CM: Small cell lung cancer: Will recent progress lead to improved outcomes? Clin Cancer Res 21: 2244-2255, 2015.

3. Seiter K: Toxicity of the topoisomerase II inhibitors. Expert Opin Drug Saf 4: 219-234, 2005.

4. Hayati F, Hossainzadeh M, Shayanpour S, Abedi-Gheshlaghi Z and Beladi Mousavi SS: Prevention of cisplatin nephrotoxicity. J Nephropharmacol 5: 57-60, 2016.

5. McKeage MJ: Lobaplatin: A new antitumour platinum drug. Expert Opin Investig Drugs 10: 119-128, 2001.

6. Harstrick A, Bokemeyer C, Scharnofkse M, Hapke G, Reile D and Schmoll HJ: Preclinical activity of a new platinum analogue, lobaplatin, in cisplatin-sensitive and -resistant human testicular, ovarian, and gastric carcinoma cell lines. Cancer Chemother Pharmacol 33: 43-47, 1993.

7. Liang Y, Sui X and Cheng Y: Clinical research progress of the new antitumor drug Lobaplatin in the treatment of small cell lung cancer. Chin J N Drugs 23: 184-188, 2014.

8. Jakupec MA, Galanski M and Keppler BK: Tumour-inhibiting platinum complexes-state of the art and future perspectives. Rev Physiol Biochem Pharmacol 146: 1-54, 2003.

9. Monneret C: Platinum anticancer drugs. From serendipity to rational design. Ann Pharm Fr 69: 286-295, 2011.

10. Fiebig HH, Henss H, von Pawel I, Gatzemeier U, Manegold CH, Edler L and Berdel W: Phase II clinical trial of lobaplatin (D-19466) in pretreated patients with small-cell lung cancer. Onkologie 19: 328-332, 1996.

11. Yang L and Qin S: Progression of Lobaplatin as the third generation platinum drug. Chin Clin Oncol 14: 1134-1139, 2009.

12. De Vore RF: Chemotherapy for small cell lung cancer. In: Pass HI, ed. Lung Cancer Principles and Practice. 2nd edition. Philadelphia, Lippincott Williams \& Wilkins, 2000.

13. Eisenhauer EA, Therasse P, Bogaerts J, Schwartz LH, Sargent D, Ford R, Dancey J, Arbuck S, Gwyther S, Mooney M, et al: New response evaluation criteria in solid tumours: Revised RECIST guideline (version 1.1). Eur J Cancer 45: 228-247, 2009.
14. Satouchi M, Kotani Y, Shibata T, Ando M, Nakagawa K, Yamamoto N, Ichinose Y, Ohe Y, Nishio M, Hida T, et al: Phase III study comparing amrubicin plus cisplatin with irinotecan plus cisplatin in the treatment of extensive-disease small-cell lung cancer: JCOG 0509. J Clin Oncol 32: 1262-1268, 2014.

15. Russell SD, Saval MA, Robbins JL, Ellestad MH, Gottlieb SS, Handberg EM, Zhou Y and Chandler B; HF-ACTION Investigators: New York Heart Association functional class predicts exercise parameters in the current era. Am Heart J 158 (4 Suppl): S24-S30, 2009.

16. Gietema JA, de Vries EG, SleijferDT, Willemse PH, Guchelaar HJ Uges DR, Aulenbacher P, Voegeli R and Mulder NH: A phase I study of 1,2-diamminomethyl-cyclobutane-platinum (II)-lactate (D-19466; lobaplatin) administered daily for 5 days. $\mathrm{Br}$ J Cancer 67: 396-401, 1993.

17. Fiebig HH, Henss H, Mross K, Meyberg F, Aulenbacher P, Burk K and Queißer W: Phase I clinical trial of lobaplatin (D-19466) after intravenous bolus injection. Onkologie 17: 142-148, 1994.

18. Basch E, Iasonos A, McDonough T, Barz A, Culkin A, Kris MG, Scher HI and Schrag D: Patient versus clinician symptom reporting using the national cancer institute common terminology criteria for adverse events: Results of a questionnaire-based study. Lancet Oncol 7: 903-909, 2006.

19. Osoba D, Rodrigues G, Myles J, Zee B and Pater J: Interpreting the significance of changes in health-related quality-of-life scores. J Clin Oncol 16: 139-144, 1998.

20. Anderson H, Hopwood P, Stephens RJ, Thatcher N, Cottier B, Nicholson M, Milroy R, Maughan TS, Falk SJ, Bond MG, et al: Gemcitabine plus best supportive care (BSC) vs BSC in inoperable non-small cell lung cancer-a randomized trial with quality of life as the primary outcome. UK NSCLC Gemcitabine Group. Non-small cell lung cancer. Br J Cancer 83: 447-453, 2000.

21. Noda K, Nishiwaki Y, Kawahara M, Negoro S, Sugiura T, Yokoyama A, Fukuoka M, Mori K, Watanabe K, Tamura T, et al: Irinotecan plus cisplatin compared with etoposide plus cisplatin for extensive small-cell lung cancer. N Engl J Med 346: 85-91, 2002.

22. Lara PN Jr, Natale R, Crowley J, Lenz HJ, Redman MW Carleton JE, Jett J, Langer CJ, Kuebler JP, Dakhil SR, et al: Phase III trial of irinotecan/cisplatin compared with etoposide/cisplatin in extensive-stage small-cell lung cancer: Clinical and pharmacogenomic results from SWOG S0124. J Clin Oncol 27: 2530-2535, 2009.

23. Jung SH, Kang SJ, McCall LM and Blumenstein B: Sample size computation for two-sample noninferiority log-rank test. J Biopharm Stat 15: 969-979, 2005.

24. Lakatos E: Sample sizes based on the log-rank statistic in complex clinical trials. Biometrics 44: 229-241, 1988.

25. Miller RP, Tadagavadi RK, Ramesh G and Reeves WB: Mechanisms of Cisplatin nephrotoxicity. Toxins (Basel) 2: 2490-2518, 2010

26. Chen MQ, Chen C, Lu HJ and Xu BH: The efficacy and toxicities of combined lobaplatin with paclitaxel as a first-line chemotherapy for advanced esophageal squamous cell carcinoma. J Thorac Dis 7: 1749-1755, 2015.

27. Yang JS, Wang T, Qiu MQ and Li QL: Comparison of efficacy and toxicity profiles between paclitaxel/lobapoatin- and cisplatin/5-fluorouracil-based concurrent chemoradiotherapy of advanced inoperable oesophageal cancer. Intern Med J 45: 757-761, 2015.

28. Li WP, Liu H, Chen L, Yao YQ and Zhao EF: A clinical comparison of lobaplatin or cisplatin with mitomycine and vincristine in treating patients with cervical squamous carcinoma. Asian Pac J Cancer Prev 16: 4629-4631, 2015.

29. Wang JQ, Wang T, Shi F, Yang YY, Su J, Chai YL and Liu Z: A randomized controlled trial comparing clinical outcomes and toxicity of lobaplatin-versus cisplatin-based concurrent chemotherapy plus radiotherapy and high-dose-rate brachytherapy for FIGO Stage II and III cervical cancer. Asian Pac J Cancer Prev 16: 5957-5961, 2015.

30. Mirsadraee S, Oswal D, Alizadeh Y, Caulo A and van Beek E Jr: The 7th lung cancer TNM classification and staging system: Review of the changes and implications. World J Radiol 4: 128-134, 2012. 